\title{
Formation and feeding of supermassive black holes in the early universe
}

\author{
Wolfgang J. Duschl ${ }^{1} \dagger$ \\ ${ }^{1}$ Institut für Theoretische Astrophysik, \\ Tiergartenstr. 15, 69121 Heidelberg, Germany \\ email: wjd@ita.uni-heidelberg.de
}

\begin{abstract}
We present a physical and numerical model of accretion driven growth of supermassive black holes. In this model we can account for $(a)$ the existence of $>10^{9} \mathrm{M}_{\odot}$ black holes already in the first quasars and (b) for the observation that the lower luminosity AGN distribution peaks later in the evolution of the Universe than that of more luminous objects.
\end{abstract}

\section{Introduction}

Currently, the most distant quasars known are located at redshifts of almost 6.5 (Fan et al. 2003). Already at this early stage of the Universe's evolution, in the center of these quasars super-massive black holes of $>10^{9} \mathrm{M}_{\odot}$ are present (Fan et al. 2001, Brandt et al. 2002a,b, Willot et al. 2003).

Moreover, the distribution of lower luminosity AGN peaks at smaller redshifts than that of more luminous AGN (Hasinger 2003, Fiore et al. 2003, Ueda et al. 2003).

\section{Physical model.}

Guided by the results of merger model calculations by Barnes \& Hernquist (1996, 1998) and Barnes (2002), we make the assumption that, due to a major merger, tidal forces have driven a large amount $\left(10^{9 \ldots 10} \mathrm{M}_{\odot}\right)$ of ISM into the central region (within a few hundred parsec of the center) of the newly formed merged galaxy. We follow the evolution of a self-gravitating accretion disk which results from the merger. It has been known for quite a while that a standard accretion disk (" $\alpha$-disk") evolves far to slowly to be of any relevance for an accretion driven growth of a central black hole (Shlosman et al. 1989, 1990). Instead, here we use the Reynolds number based viscosity prescription proposed by Duschl et al. (1998, 2000) and Richard \& Zahn (1999). We allow also for advection dominated accretion (Beckert \& Duschl 2002).

\section{Accretion disk evolution and black hole growth.}

We performed numerical model calculations and present a typical example in Fig. 1. The initial disk mass is $10^{10} \mathrm{M}_{\odot}$ and the viscosity paramter $\beta=10^{-3}$. For numerical convenience we assumed a seed black hole of $10^{3} \mathrm{M}_{\odot}$. The final black hole mass is determined mainly by the length of an initial Eddington limited accretion phase. We find that this type of accretion disk allows for a sufficiently fast growth of a super-massive black

$\dagger$ Work supported by the Deutsche Forschungsgemeinschaft (DFG) through the Sonderforschungsbereich "Galaxies in the Young Universe" (SFB 439) 


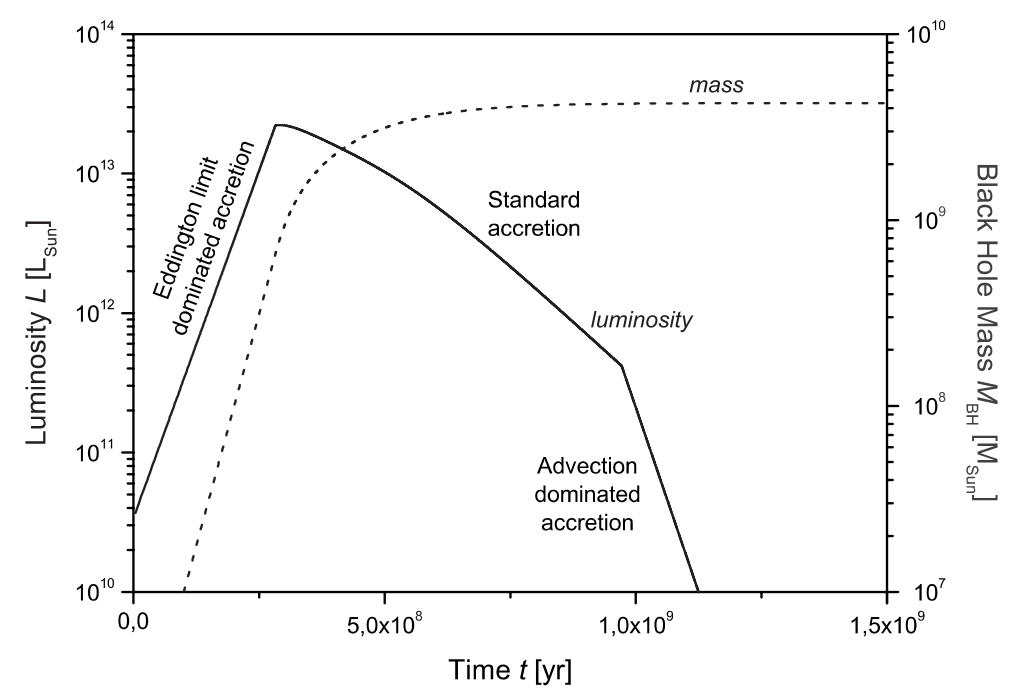

Figure 1. Evolution of luminosity (full line) and black hole mass (broken line) as a function of time. For the disk parameters, see the text.

hole. The high-luminosity phase ends rather abruptly when radial advection of energy becomes important, limiting the high-luminosity phase to some $10^{8}$ years.

We find an inverse correlation between the peak activity of a galactic center and the time scale it takes to achieve it: The lower the peak luminosity is, the longer it takes to reach it.

A much more detailled account of this work will be presented in Duschl \& Strittmatter (2004, in prep.).

\section{References}

Barnes, J. E. 2002, MNRAS, 333, 481

Barnes, J. E., \& Hernquist, L. 1996, ApJ, 471, 115

Barnes, J. E., \& Hernquist, L. 1998, ApJ, 495, 187

Beckert, T., \& Duschl, W. J. 2002, A\&A, 387, 422

Brandt, W. N., et al. 2002a, ApJ, 569, L5

Brandt, W. N., et al. 2002b, MPE-Report, 279, 235

Duschl, W. J., Strittmatter, P. A., \& Biermann, P. L. 1998, BAAS, 30, 917

Duschl, W. J., Strittmatter, P. A., \& Biermann, P. L. 2000, A\&A, 357, 1123

Fan, X., et al. 2001, AJ, 122, 2833

Fan, X., et al. 2003, AJ, 125, 1649

Fiore, F., et al. 2003, A\&A, 409, 79

Hasinger, G. 2003, AIP Conf. Procs., 666, 227

Richard, D., \& Zahn, J.-P. 1999, A\&A, 347, 734

Shlosman, I., Begelman, M. C., \& Frank, J. 1990, Nature, 345, 679

Shlosman, I., Frank, J., \& Begelman, M. C. 1989, Nature, 338, 45

Ueda, Y., Akiyama, M., Ohta, K., \& Miyaji, T. 2003, ApJ, 598, 886

Willot, C. J. McLure, R. J., \& Jarvis, M. J. 2003, ApJ, 587, L15 